\title{
Etched LPFGs in Reflective Configuration for Sensitivity and Attenuation Band Depth Increase
}

\author{
Ignacio Del Villar, Abian B. Socorro, Jesus M. Corres, Ignacio R. Matias, Senior Member, IEEE, \\ Jose Luis Cruz, and Gaspar Rego
}

\begin{abstract}
A reflection configuration setup for long-period fiber gratings is presented. It permits to obtain a unique band with attenuation double than that obtained in transmission configuration, which is interesting for applications where this value is reduced (e.g., the mode transition phenomenon). The method is based on the deposition of a silver mirror at the end of the optical fiber, which permits to absorb the power transmitted through cladding modes and to avoid the generation of interferometric bands. The method also solves the requirement of a precise cleave or to polish the end of the grating, a drawback present in other publications. The versatility of the setup has been proved by application of the cladding etching technique until the attenuation band corresponding with the first guided mode in the cladding is visualized in an optical spectrum analyzer. The experimental results are supported by the numerical data obtained with a method based on the exact calculation of core and cladding modes and the utilization of coupled mode theory.
\end{abstract}

Index Terms-Optical fiber, gratings, optical fiber transducers, reflection configuration.

\section{INTRODUCTION}

$\mathbf{L}$ ONG period fiber gratings (LPFGs) consist of a periodic index modulation of the core refractive index in a single mode fiber (SMF) with a much longer period than the optical wavelength. This enables a coupling between the core mode and co-propagating cladding modes. Each coupling of light transmitted through the core to a specific cladding mode leads to the generation of an attenuation band in the transmission spectrum, which can be used in optical communications, laser technology and sensors.

The first publication showing this type of device dates from 1986 [1] and the integration in the fiber by UV-photoinscripted was demonstrated in 1996 [2]. Thereafter, LPFGs have been vastly used. Also in 1996 another work was published showing one of the major fields of application of LPFGs: since there

Manuscript received December 4, 2015; revised January 18, 2016; accepted February 10, 2016. Date of publication March 1, 2016; date of current version March 29, 2016. This work was supported by the Spanish Ministry of Education under Grant FEDER TEC2013-43679-R and Grant FEDER TEC2013-46643-C2-1-R.

I. Del Villar, A. B. Socorro, J. M. Corres, and I. R. Matias are with the Electrical and Electronic Engineering Department, Public University of Navarra, Pamplona 31006, Spain (e-mail: ignacio.delvillar@unavarra.es; ab.socorro@unavarra.es; jmcorres@unavarra.es; natxo@unavarra.es).

J. L. Cruz is with the Department of Applied Physics and Electromagnetism, University of Valencia, Burjassot 46100, Spain (e-mail: jose.l.cruz@uv.es).

G. Rego is with Instituto de Engenharia de Sistemas e Computadores, Tecnologia e Ciência, Porto 4169-007, Portugal, and also with Escola Superior de Tecnologia e Gestão-Instituto Politécnico de Viana do Castelo, Viana do Castelo 4900-348, Portugal (e-mail: gmrego@fc.up.pt).

This paper has supplementary downloadable material at http://ieeexplore.ieee.org, provided by the authors.

Color versions of one or more of the figures in this letter are available online at http://ieeexplore.ieee.org.

Digital Object Identifier 10.1109/LPT.2016.2529701 is an interaction with the cladding modes, and the cladding is surrounding by the external medium, they can be used as sensors [3].

This last domain has found more interest in the scientific community due to several important contributions that have permitted to increase the sensitivity of LPFGs. The first one is the discovery of the turn dispersion point (TDP), a phenomenon that occurs typically for higher order cladding modes [4]. The second one is the mode transition (MT) [5], [6], which occurs when a thin-film is deposited on the cladding of the LPFG [7], [8]. This permits to obtain sensors with an increased sensitivity to refractive index and to chemical or biological species, if the thin-film is sensitive to them [9], [10]. The combination of the TDP with the MT has permitted to achieve a record sensitivity of $9900 \mathrm{~nm}$ per refractive index unit (nm/RIU) [11].

A last way to increase the sensitivity of LPFGs is the reduction of the cladding diameter (i.e. by tapering or etching the fiber) [12], [13]. In a recent work it has been theoretically proved that $143000 \mathrm{~nm} / \mathrm{RIU}$ can be attained by combining adequately the three phenomena explained and by focusing $\mathrm{pm}$ the attenuation band that corresponds with the lowest order mode guided in the optical fiber cladding [14].

Most of the contributions on LPFGs are focused on a transmission configuration. However, there are some works where a reflective configuration is used [10], [15], [16], which permits to obtain a small sensor head that can be used to measure low volumes of chemical or biological samples. The problem when using a reflective configuration is that it leads to the generation of non-desired fringes that overlap the LPFG attenuation bands [10]. In order to obtain a single attenuation band, a precise cleave at the end of LPFG or polishing after cleaving the optical fiber is required [15], [16].

This letter presents a simpler method that avoids the need of a polishing or a precise position of the fiber cleave. Moreover, with this configuration it is possible to increase the depth of the attenuation band, which is interesting to prevent the fading of the dips at the mode transition [17]. In order to prove the versatility of this setup, a sensitivity enhancement has been explored, using the cladding etching technique. A 5-fold increase in the sensitivity to refractive index has been attained by etching until the attenuation band corresponding to lowest order mode guided in the optical fiber cladding is visualized in the optical spectrum analyzer.

\section{EXPERIMENTAL SETUP}

In Fig. 1a the reflection configuration setup is depicted. It consists of a broadband source (Agilent 83437A) that 


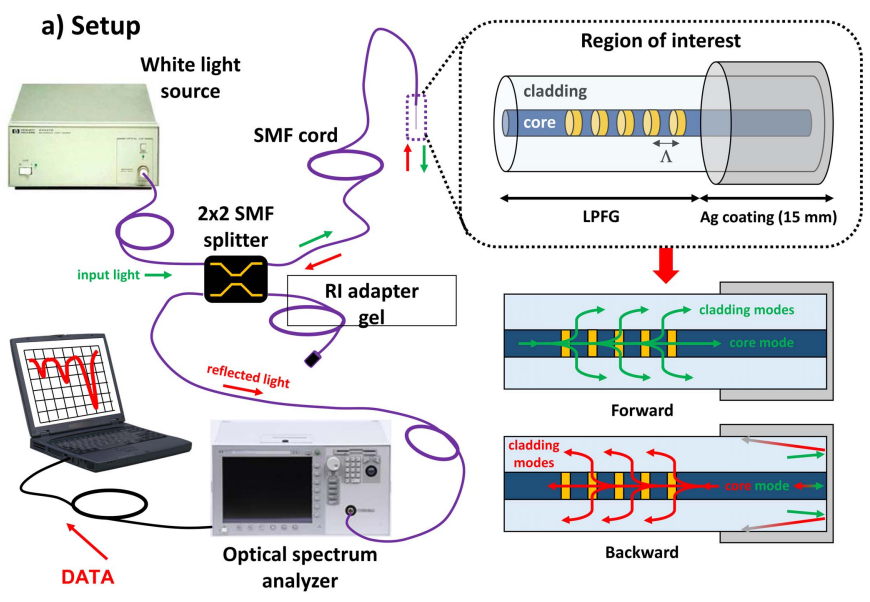

b) Reflection spectra

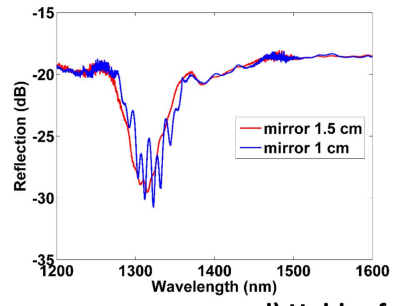

d) Holder for etching

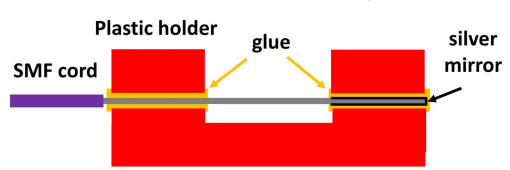

Fig. 1. a) Reflection-configuration experimental setup: light is reflected but light cladding modes are absorbed in the silver layer; b) Reflection spectra for two silver coating lengths; c) Optical power at wavelength $1550 \mathrm{~nm}$ during the silver mirror creation; d) Holder used for etching the sensor head.

launches light into one end of an SMF $2 \times 2$ coupler. The second end is connected to an Agilent 86140B optical spectrum analyzer which monitors the reflection spectrum. The third end is connected to the sensing pigtail where the LPFG is written. The last end is surrounded with an index matching gel.

For the sensing pigtail, a commercial LPFG from O/E Land was used. A grating with period $200 \mu \mathrm{m}$ and length $25 \mathrm{~mm}$ was written in a HI1060 Corning single mode fiber (core diameter $5 \mu \mathrm{m}$, cladding diameter $125 \mu \mathrm{m}$ and cladding refractive index that of silica [18]). The surrounding medium refractive index (SRI) was air (refractive index 1).

After that, the LPFG was cleaved at two centimeters of the grating. Then $1.5 \mathrm{~cm}$ of the fiber were immersed in a solution where a reaction for obtaining a silver mirror was developed with the Tollen's reagent method [10]. For a lower length fringes appear in the optical spectrum (see Fig. 1b). In order to know when the mirror has been fabricated on the fiber head, the optical power is tracked at wavelength $1550 \mathrm{~nm}$ (see Fig. 1c) and the deposition is stopped when the signal is stabilized. The silver coating acts as a mirror for the core mode at the same time the cladding modes are absorbed.

\section{THEORY}

In order to analyze the response of the device described before, the vectorial method described in [19] is used. It is an expansion of the coupled mode method of Erdogan [20] for a multilayer structure and it follows the same steps indicated in [21], but hybrid modes instead of LP modes are used in order to improve the simulation. In [19] it was explained that, for specific cases (for instance the deposition of high refractive index thin-films), the LP mode approximation is not valid. Consequently, for the sake of accuracy this vectorial method based on the exact solutions of Maxwell equations is used.

In order to obtain the transmitted power, the couple-mode equations are necessary [20], [21]:

$$
\begin{array}{r}
\frac{d F_{1 k}(z)}{d z}=-j \sum_{j=1}^{M} K_{1 j, 1 k} F_{1 j}(z) \exp \left(-j\left(\beta_{1 j}-\beta_{1 k}\right) z\right) \frac{d F_{1 k}(z)}{d z} \\
\text { for } k=1,2, \ldots, M
\end{array}
$$

where $F_{1 k}$ is the complex amplitude of each propagating mode, $K_{1 j, 1 k}$ is the coupling coefficient between modes $\mathrm{j}$ and k, $\beta_{1 j}$ and $\beta_{1 k}$ are the propagation constants of $j$ and $k$ modes respectively, and $M$ is the number of modes analyzed.

The transmitted power at the end of the LPFG can be expressed as:

$$
\frac{\left|F_{11}(L)\right|^{2}}{\left|F_{11}(0)\right|^{2}}
$$

In this letter, a reflective configuration is used (see Fig. 1). Consequently, light is transmitted through the grating two times. Since the cladding modes are absorbed by the silver coating, it is not necessary to consider the phase of the modes transmitted in the core and in the cladding. Hence, the reflected power can be expressed as:

$$
\left(\frac{\left|F_{11}(L)\right|^{2}}{\left|F_{11}(0)\right|^{2}}\right)^{2}
$$

In those cases where only the analysis of wavelength is required, the modified phase matching condition is used [21]:

$$
\beta_{11}(\lambda)+s_{0} \zeta_{11,11}(\lambda)-\left(\beta_{1 j}(\lambda)+s_{0} \zeta_{1 j, 1 j}(\lambda)\right)=\frac{2 \pi N}{\Lambda}
$$

where $\beta_{11}$ and $\beta_{1 j}$ are the propagation constants of the core and the $\mathrm{j}$ cladding modes respectively, $\Lambda$ is the period of the grating, $\zeta_{11,11}$ and $\zeta_{1 j, 1 j}$ are the self-coupling coefficients of the core and the $\mathrm{j}$ cladding modes, $s_{0}$ is the coefficient of the first Fourier component of the grating and $N$ the diffraction order.

The notation used for the modes is: $\mathrm{HE}_{1,1}$ for the core mode; $\mathrm{HE}_{1,2}$ for the first $\mathrm{HE}_{1, \mathrm{j}}$ cladding mode, $\mathrm{HE}_{1,4}$ for the second $\mathrm{HE}_{1, \mathrm{j}}$ cladding mode, and so on; $\mathrm{EH}_{1,3}$ for the first $\mathrm{EH}_{1, \mathrm{j}}$ cladding mode, $\mathrm{EH}_{1,5}$ for the second $\mathrm{EH}_{1, \mathrm{j}}$ cladding mode, and so on [20], This notation will serve to identify the attenuation bands in the optical spectrum.

\section{RESUlTS}

The theoretical and experimental transmission and reflection spectra of the LPFG depicted in Fig. 1 can be observed in Fig. 2 and Fig. 3, respectively. The depth of the attenuation bands is increased by a factor of 2 with the reflection configuration (the scale of the y axis is double for the reflection spectra). This is caused by the discrepancy between expressions 2 

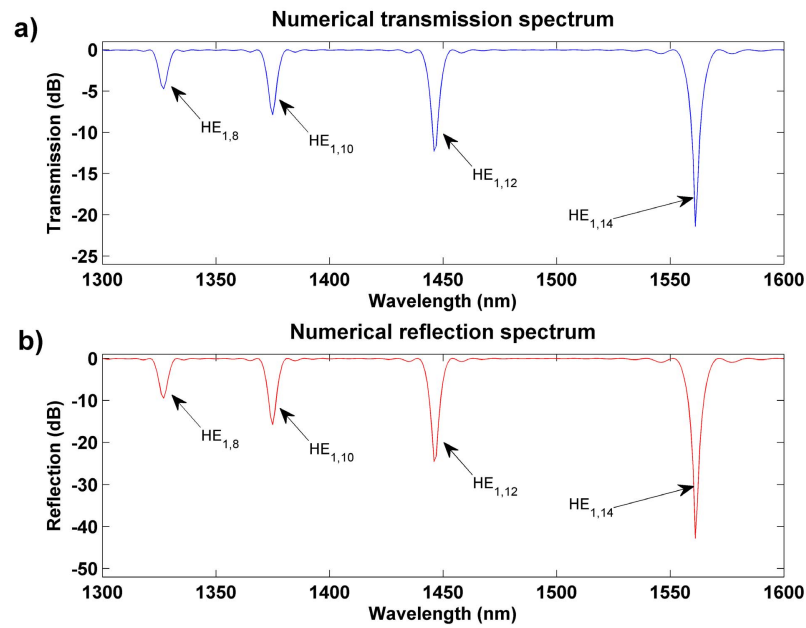

Fig. 2. Numerical spectra for an LPFG with period $200 \mu \mathrm{m}$ and surrounding medium refractive index 1: a) transmission configuration; b) reflection configuration.

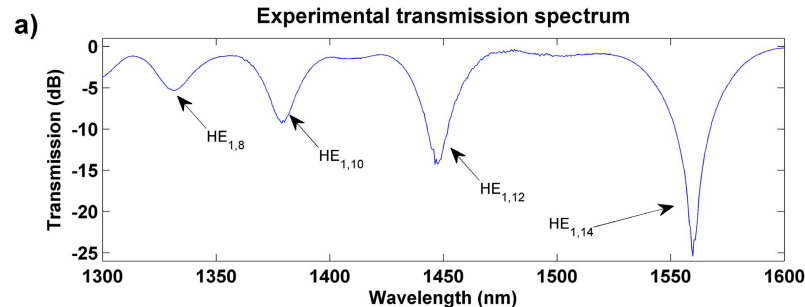

b)

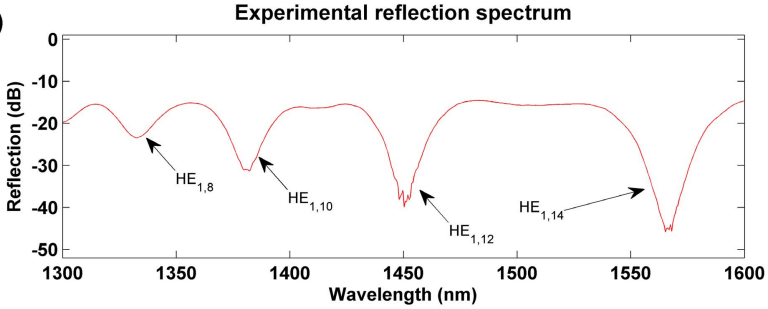

Fig. 3. Experimental spectra for an LPFG with period $200 \mu \mathrm{m}$ and surrounding medium refractive index 1: a) transmission configuration; b) reflection configuration.

and 3. Only the $\mathrm{HE}_{1,14}$ band does not satisfy this condition because the OSA sensitivity limit $(-90 \mathrm{dBm}$ in the range 1250 $1650 \mathrm{~nm}$ ) has been overcome in the experimental graphs.

Gratings in thinned fibers are more sensitive to the external refractive index because the electromagnetic field is less confined in the glass of the cladding. Consequently, the propagation factor of the cladding mode has an enhanced dependence on the surrounding material.

To support this idea the resonances of a grating were calculated as a function of the grating pitch. Fig. 4a compares the results for a standard fiber surrounded by air (refractive index 1) and for a fiber embedded in a refractive index of 1.321. Fig. 4b shows the results for a fiber having a diameter reduced to $27 \mu \mathrm{m}$. The thinner fiber supports only one cladding mode with rotational symmetry in the range of wavelengths considered in this simulation, and it can be clearly observed that this mode has more sensitivity to the external refractive index than any of the modes of the pristine fiber.

For the sake of comparison, the bands $\mathrm{HE}_{1,10}$ and $\mathrm{HE}_{1,12}$ of the unetched LPFG, which are located closer to the $\mathrm{HE}_{1,2}$ a)

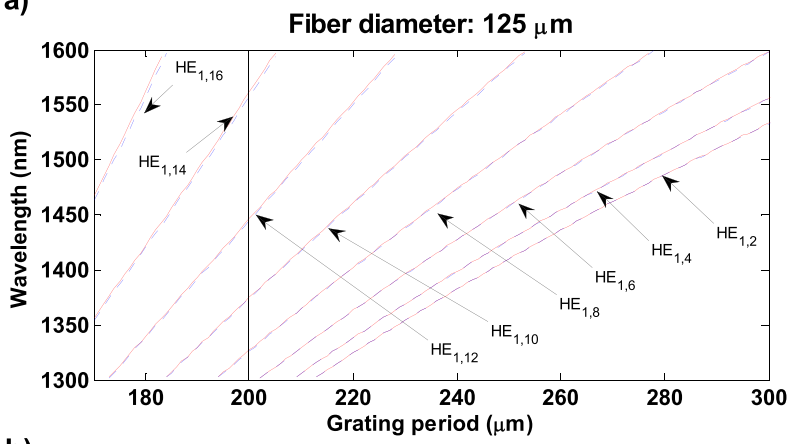

b)

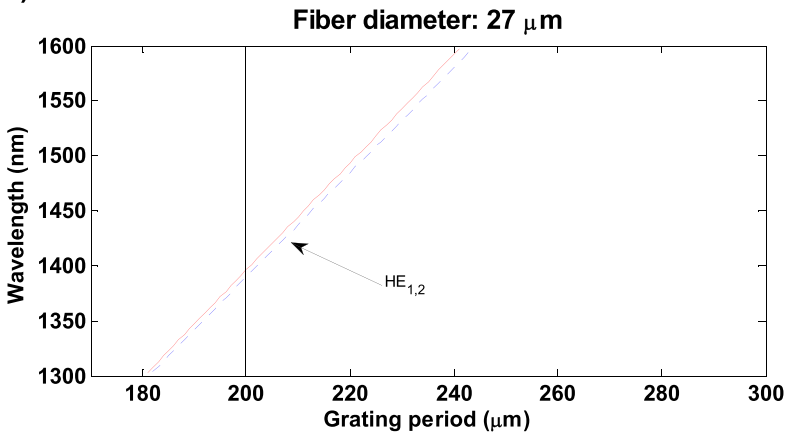

Fig. 4. Variation of mode resonance wavelength with LPFG period for a) cladding diameter $125 \mu \mathrm{m}$; b) cladding diameter $27 \mu \mathrm{m}$. Surrounding medium refractive index: 1 for solid lines and 1.321 for dotted lines.

band of the etched LPFG, experiment wavelength shifts from 1375 to $1373.8 \mathrm{~nm}(1.2 \mathrm{~nm})$, and from 1446.4 to $1444.6 \mathrm{~nm}$ $(1.8 \mathrm{~nm})$ respectively, whereas $\mathrm{HE}_{1,2}$ band shifts from 1396 to $1390 \mathrm{~nm}(6 \mathrm{~nm})$. Taking the average value of the wavelength shift of $\mathrm{HE}_{1,10}$ and $\mathrm{HE}_{1,12}$ bands, there is a 4-fold increase in sensitivity.

In order to prove the ability of this sensor to support the cladding etching technique, the silver layer was covered with a glue that avoids the degradation of the silver mirror. For an etching up to around $100 \mu \mathrm{m}$ [12], no holder is required to maintain the LPFG straight. However, if we are interested in a highest sensitivity in the device, the attenuation band corresponding with the lowest order cladding mode must be monitored. This leads to a very narrow structure sensitive to vibrations. Hence the plastic structures depicted in Fig 1d is necessary.

Then the sensor was immersed in a $40 \%$ hydrofluoric acid (HF) solution and the etching was stopped, according to [14], when only the attenuation band belonging the lowest order cladding mode was visible (i.e. $\mathrm{HE}_{1,2}$ ).

The experimental results are shown in Fig. 5. The wavelength shift obtained by immersion in water (refractive index 1.321 [22]) of the original LPFG is compared to the wavelength shift obtained with the cladding etched LPFG. The bands $\mathrm{HE}_{1,10}$ and $\mathrm{HE}_{1,12}$ of the unetched LPFG experiment wavelength shifts from 1381.4 to $1379.8 \mathrm{~nm}(1.6 \mathrm{~nm})$, and from 1551 to $1449 \mathrm{~nm}(2 \mathrm{~nm})$ respectively, whereas the $\mathrm{HE}_{1,2}$ band of the etched LPFG shifts from 1397 to $1387 \mathrm{~nm}$ $(10 \mathrm{~nm})$. This indicates that a 5-fold sensitivity increase is attainted, confirming the theoretical analysis. The attenuation band $\mathrm{HE}_{1,2}$ of the etched LPFG was obtained by tracking the 

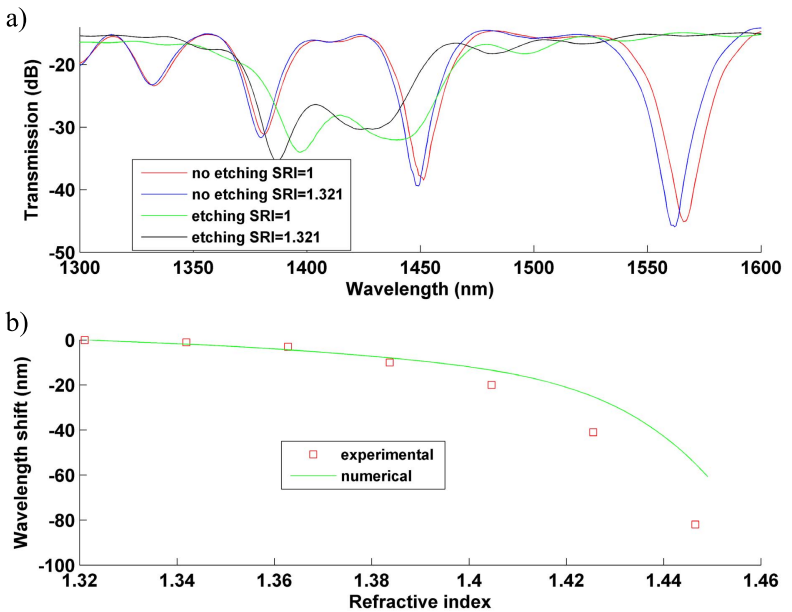

Fig. 5. a) Reflection spectra for an LPFG with period $200 \mu \mathrm{m}$ and the same LPFG etched until the lowers order band is visible. Two refractive indices are analyzed: 1 and 1.321 b) Sensitivity to refractive index of the etched LPFG.

optical spectrum until only the band located at the shortest wavelength was visible, according to the numerical study shown in the video 1 offered as supplementary material. $\mathrm{HE}_{1,2}$ band is splitted in two bands due to the change in the coupling conditions due to the reduction of the cladding diameter, which causes an overcoupling [23]. This effect can be observed also in the simulation of the evolution of the attenuation bands as the cladding refractive index is reduced (video 1 in the supplementary material). In order to avoid this wide spectrum an LPFG with a lower length should be used, which would permit to obtain a shorter device.

In Fig. 5b the sensitivity (wavelength shift versus refractive index) of the etched fiber is analyzed theoretically and experimentally showing a value of $653 \mathrm{~nm} / \mathrm{RIU}$. The mismatch at refractive indices approaching the cladding is due to the utilization of silica refractive index model [18], which is not exactly the same as the HI0160 Corning fiber.

\section{CONCLusions}

To conclude, in this letter it has been proved that the application of a silver mirror, which covers also part of the cladding in an LPFG, permits to obtain a single attenuation band in the optical spectrum. The band depth is increased, which permits to reduce the fading effect in the attenuation band that occurs with thin-film coated LPFGs in the mode transition.

The method is quite simple and does not require polishing or cleaving the optical fiber at a specific point. Moreover, the device can be used in two configurations. The mirrored region can be used to develop a holder that protects the device when etched up to a diameter where the device is sensitive to vibrations. This opens the path towards the study LPFGs with optimized period where the sensitivity record indicated in [14] can be attained. However, a lower etching can be performed in order to avoid the necessity of a holder structure. The device will be less sensitive but can be introduced in small cuvettes, which is interesting for chemical or biological applications.

\section{REFERENCES}

[1] J. N. Blake, B. Y. Kim, and H. J. Shaw, "Fiber-optic modal coupler using periodic microbending," Opt. Lett., vol. 11, no. 3, pp. 177-179, 1986.

[2] A. M. Vengsarkar, P. J. Lemaire, J. B. Judkins, V. Bhatia, T. Erdogan, and J. E. Sipe, "Long-period fiber gratings as band-rejection filters," J. Lightw. Technol., vol. 14, no. 1, pp. 58-65, Jan. 1996.

[3] V. Bhatia and A. M. Vengsarkar, "Optical fiber long-period grating sensors," Opt. Lett., vol. 21, no. 9, pp. 692-694, 1996.

[4] X. Shu, L. Zhang, and I. Bennion, "Sensitivity characteristics of longperiod fiber gratings," J. Lightw. Technol., vol. 20, no. 2, pp. 255-266, Feb. 2002.

[5] I. Del Villar, I. R. Matías, F. J. Arregui, and P. Lalanne, "Optimization of sensitivity in Long Period Fiber Gratings with overlay deposition," Opt. Exp., vol. 13, no. 1, pp. 56-69, 2005.

[6] A. Cusano et al., "Mode transition in high refractive index coated long period gratings," Opt. Exp., vol. 14, no. 1, pp. 19-34, 2006.

[7] N. D. Rees, S. W. James, R. P. Tatam, and G. J. Ashwell, "Optical fiber long-period gratings with Langmuir-Blodgett thin-film overlays," Opt. Lett., vol. 27, no. 9, pp. 686-688, 2002.

[8] Z. Wang, J. R. Heflin, R. H. Stolen, and S. Ramachandran, "Analysis of optical response of long period fiber gratings to nm-thick thin-film coatings," Opt. Exp., vol. 13, no. 8, pp. 2808-2813, 2005.

[9] P. Pilla et al., "Optical chemo-sensor based on long period gratings coated with $\delta$ form syndiotactic polystyrene," IEEE Photon. Technol. Lett., vol. 17, no. 8, pp. 1713-1715, Aug. 2005.

[10] D. W. Kim, Y. Zhang, K. L. Cooper, and A. Wang, "In-fiber reflection mode interferometer based on a long-period grating for external refractive-index measurement," Appl. Opt., vol. 44, no. 26, pp. 5368-5373, 2005.

[11] P. Pilla, C. Trono, F. Baldini, F. Chiavaioli, M. Giordano, and A. Cusano, "Giant sensitivity of long period gratings in transition mode near the dispersion turning point: An integrated design approach," Opt. Lett., vol. 37, no. 19, pp. 4152-4154, 2012.

[12] X. Chen, K. Zhou, L. Zhang, and L. Bennion, "Dual-peak long-period fiber gratings with enhanced refractive index sensitivity by finely tailored mode dispersion that uses the light cladding etching technique," Appl. Opt., vol. 46, no. 4, pp. 451-455, Feb. 2007.

[13] M. Śmietana, M. Koba, P. Mikulic, and W. J. Bock, "Measurements of reactive ion etching process effect using long-period fiber gratings," Opt. Exp., vol. 22, no. 5, pp. 5988-5994, 2014.

[14] I. Del Villar, "Ultrahigh-sensitivity sensors based on thin-film coated long period gratings with reduced diameter, in transition mode and near the dispersion turning point," Opt. Exp., vol. 23, no. 7, pp. 8389-8398, Apr. 2015

[15] G. Quero et al., "Reflection-type long period grating biosensor for detection of drug resistant bacteria: The OptoBacteria project," in Proc 18th AISEM Annu. Conf., Feb. 2015, pp. 1-4.

[16] J. Huang, X. Lan, A. Kaur, H. Wang, L. Yuan, and H. Xiao, "Reflectionbased phase-shifted long period fiber grating for simultaneous measurement of temperature and refractive index," Opt. Eng., vol. 52, no. 1, p. 014404, 2013.

[17] I. Del Villar, I. R. Matías, F. J. Arregui, and M. Achaerandio, "Nanodeposition of materials with complex refractive index in long-period fiber gratings," J. Lightw. Technol., vol. 23, no. 12, pp. 4192-4199, Dec. 2005.

[18] I. H. Malitson, "Interspecimen comparison of the refractive index of fused silica," J. Opt. Soc. Amer., vol. 55, no. 10, pp. 1205-1208, 1965.

[19] I. Del Villar, I. R. Matías, and F. J. Arregui, "Influence on cladding mode distribution of overlay deposition on long-period fiber gratings," J. Opt. Soc. Amer. A, vol. 23, no. 3, pp. 651-658, 2006.

[20] T. Erdogan, "Cladding-mode resonances in short- and long-period fiber grating filters," J. Opt. Soc. Amer. A, vol. 14, no. 8, pp. 1760-1773, 1997.

[21] E. Anemogiannis, E. N. Glytsis, and T. K. Gaylord, "Transmission characteristics of long-period fiber gratings having arbitrary azimuthal/radial refractive index variations," J. Lightw. Technol., vol. 21, no. 1, pp. 218-227, Jan. 2003.

[22] M. Daimon and A. Masumura, "Measurement of the refractive index of distilled water from the near-infrared region to the ultraviolet region," Appl. Opt., vol. 46, no. 18, pp. 3811-3820, May 2007.

[23] T. Erdogan, "Fiber grating spectra," J. Lightw. Technol., vol. 15, no. 8, pp. 1277-1294, Aug. 1997. 